\title{
Sistema de la calidad en los laboratorios de ensayos
}

\section{Gustavo Delgado ${ }^{*}$}

1. Universidad Nacional Autónoma de Nicaragua, León (UNAN-LEÓN). Facultad de Ciencias y Tecnología, Departamento de Química, Laboratorio de Análisis de Trazas de Metales Pesados (LATMP), Edificio de Ciencias Básicas, León, Nicaragua. Tel: (505) 2311 5013, ext: 1132. Fax: (505) 23114012.

\section{RESUMEN}

El presente artículo tiene como objetivo describir los componentes básicos del sistema de la calidad para la competencia técnica de los laboratorios de ensayos basado en la norma ISO/IEC 17025:2005. Se describen los diferentes requisitos que los laboratorios de ensayos deben cumplir para garantizar que los resultados de los análisis tengan trazabilidad, precisión y confiabilidad. Se proponen metas y estrategias básicas que el laboratorio debe implementar basado en una organización bien definida cuyas actividades deben estar fundamentadas en procedimientos documentados, validados y equipos calibrados. Se detallan en forma condensada los elementos básicos de la norma, tratando de presentar en forma simple el amplio espectro de los requisitos de gestión y de los requisitos técnicos necesarios para brindar servicios de calidad a la comunidad y sociedad en general.

Palabras claves: Sistema de la calidad en laboratorios, requisitos para la competencia de laboratorios de ensayos.

\section{INTRODUCCIÓN}

Tradicionalmente, los clientes confiaban en el proceso de inspección que las empresas productivas llevaban a cabo para controlar la calidad del producto antes de ser consumido o en las referencias de los usuarios antes de seleccionar y hacer uso de un servicio. Pero, la inspección no es una garantía de que el producto tendrá la aptitud para el uso previsto durante su vida útil o que el servicio cumplirá con lo esperado por el cliente.

Deming, en uno de sus catorce puntos, establece que hay que eliminar la dependencia de la inspección en masa ${ }^{[1]}$, como única actividad para la gestión de la calidad. Es necesaria una gestión más integral de la calidad, donde se enfoque en todo el proceso y en la satisfacción constante del cliente. Por otra parte, si tenemos en cuenta que los clientes de todo el mundo y en todas las épocas han exigido, exigen y seguirán exigiendo la garantía de que el producto o servicio por el que están pagando se ajuste a sus especificaciones (expectativas) y funcione como ellos esperan, la inspección como único elemento de control no es un respaldo de la calidad.

La única forma de lograr que cualquier producto elaborado o servicio prestado por una organización (empresa, laboratorio o institución), cumpla con la calidad esperada, es que dicha organización posea un sistema adecuado y eficaz de gestión de la calidad. Desde la adopción de la calidad total por la industria japonesa en los años sesenta y desde los ochenta en los Estados Unidos, la gestión de la calidad, se ha convertido actualmente en un "modus vivendi". La globalización de los mercados impone normas internacionales que ponen en juego la competitividad de las empresas o laboratorios. El dilema no debe ir más allá de si una organización podría cumplir o continuar con las prácticas de la Gestión de la Calidad Total (TQM); la interrogante es cómo implementarlas mejor y hacerlas sostenibles.

La supervivencia de una organización moderna dependerá de su capacidad de mantener la constancia en la calidad. De acuerdo a Edward Deming, 'La ley de sobrevivencia de Charles Darwin en la adaptación de la libre empresa es como la solución natural. Los únicos sobrevivientes serán las compañías con constancia en el propósito de lograr la calidad, productividad y servicios'.

Los padres de la calidad ${ }^{[1-4]}$ han formulado elementos claves para implementar el sistema de gestión de la calidad. Así por ejemplo, Edward Deming estableció tres elementos básicos de la calidad: apropiación (disfrutar del trabajo), innovación (creatividad) y cooperación (trabajo en equipo); Joseph M. Juran destacó la planificación de la calidad, la mejora continua y el control de la calidad; y Kaouru Ishikawa, destacó los siguientes puntos: la simplificación de la estadística del control de calidad, la utilización de las cartas de control y la gestión de la calidad en todos los niveles de la empresa, a la cual se le denomina Gestión de la Calidad Total o sus siglas en inglés TQM (Total Quality Management). 
Los laboratorios de ensayo de la Universidad Nacional Autónoma de Nicaragua, León (UNAN-León) no están excluidos a la filosofía de la Calidad Total. Existen dos tipos de normas por las cuales un laboratorio se puede regir: las norma ISO $9001: 2008^{[5]}$ y la ISO/IEC $17025: 2005^{[6]}$.

El primero se enfoca al Sistema de Gestión basada en el esquema de proceso ${ }^{[5,7]}$, donde se destaca el ciclo que va desde la responsabilidad de la Dirección, la gestión de los recursos, el proceso de transformación, análisis y mejora, hasta la satisfacción del cliente. En tanto que la norma ISO/IEC 17025:2005, se enfoca en la competencia técnica que tienen los laboratorios para realizar ensayos y brindar a sus clientes resultados de calidad: exactos, precisos y confiables. Este artículo pretende describir los principios básicos de la calidad basado en competencia de los requisitos establecidos por la norma ISO/IEC 17025:2005.

\section{LA CALIDAD DEL LABORATORIO Y EL CLIENTE}

Cómo funciona el Sistema de Gestión de la Calidad de un laboratorio de ensayo dentro de una corporación (la UNAN-León en nuestro caso). El laboratorio se considera como un subconjunto. La corporación puede tener implantada un sistema ISO $9001: 08^{[7]}$ u otra norma similar, pero puede ser que el laboratorio no cumpla con la competencia técnica para la realización de sus ensayos basada en la ISO/IEC 17025:2005. En estos casos el laboratorio debe satisfacer a dos tipos de clientes: INTERNOS (investigadores de otras unidades) y EXTERNOS (usuarios que no pertenecen a la corporación). El laboratorio juega un papel crucial en cuanto a la satisfacción del cliente. En muchos casos los clientes internos son más exigentes que los externos. Veamos el ejemplo de una empresa productiva.

Pensemos en la función de un laboratorio experimental como unidad de control de calidad y que presenta los siguientes casos:

- La planta productiva produce un producto conforme a las especificaciones establecidas en la empresa, pero el laboratorio analítico lo define como no conforme. La planta debe revisar los controles de calidad durante el proceso y si verifica alguna anomalía, debe rehacer el producto. Pero si todos los controles del proceso están dentro de los límites establecidos, el laboratorio debe revisar su proceso analítico y corregir las anomalías encontradas para satisfacer a su cliente interno.

- Si el laboratorio certifica que un producto es conforme (dentro de la especificación) estando realmente fuera de la especificación, la situación se vuelve difícil para el laboratorio, puesto que generalmente el cliente interno confía en los resultados, pero el cliente externo, quien es el que consume el producto, manifestará su insatisfacción y perderá la confianza en la calidad de la empresa. Esto conlleva una dismunución en la demanda y por ende al desprestigio o probable desaparición de la empresa. El cliente espera datos útiles, consistentes, exactos y oportunos. Un laboratorio debe demostrar a sus clientes que se preocupa por sus éxitos. Si las expectativas del cliente no se cumplen y si los resultados suministrados por el laboratorio son de vital importancia, este cliente perderá la confianza en el laboratorio.

Se puede hacer una comparación entre una industria y un laboratorio. En la planta manufacturera se fabrican productos para sus clientes y el laboratorio produce datos para sus clientes. No puede existir diferencia en las calidades de los productos. La planta produce productos tangibles y el laboratorio produce productos intangibles.

\section{DESCRIPCIÓN GENERAL DE LOS REQUISITOS DE LA CALIDAD}

La norma ISO/IEC 17025:2005, establece dos tipos de requisitos que tienen que cumplir los laboratorios de ensayos para demostrar su competencia técnica. Estos requisitos son: requisitos relativos a la gestión y los requisitos técnicos. A continuación se discuten los componentes básicos de estos requisitos. La descripción no corresponde al orden establecido en la norma. El autor realiza la descripción e interpretaciones de los requisitos de acuerdo a la experiencia de su implementación en los laboratorios de ensayos y establece la diferencia entre la certificación y la acreditación.

\subsection{La Organización}

El laboratorio debe ser una entidad legal, con una organización bien definida y con el personal directivo clave y sus funciones claramente definidas. Debe tener un director que tenga autoridad y poder de decisión para garantizar la eficacia del funcionamiento del laboratorio, un responsable de la calidad que garantice la calidad a todos los niveles del laboratorio, y un director técnico que asegure los recursos necesarios para llevar a cabo eficazmente la realización de los ensayos con sus controles de calidad.

Cuando el laboratorio es pequeño, una misma persona puede desempeñar varios cargos. Por ejemplo, el Director del laboratorio puede hacerse cargo de 
la dirección técnica y puede eventualmente ser analista. La autoridad máxima del laboratorio debe comprometerse con la calidad a través de la declaración de una política de la calidad, donde se destaquen la visión y un plan estratégico para la búsqueda constante de la excelencia, con sus metas y objetivos, que deben aplicarse a todos los niveles de la empresa.

A continuación se detallan algunas metas y estrategias fundamentales de la calidad que debe implementar un laboratorio.

\section{Metas}

- Ser el mejor en calidad de sus servicios.

- Conseguir la mejor calidad a través del mejoramiento continuo.

- Enviar a tiempo los resultados de los ensayos que reúnan las expectativas del cliente y que infunda confianza en la puntualidad del laboratorio y la evidencia objetiva por su dedicación a la calidad.

\section{Estrategias}

- Efectuar el análisis correcto desde la primera vez; eliminar los análisis repetidos y la información falsa.

- Mantener y operar el laboratorio en condiciones de salud e higiene ocupacional seguro.

- Utilizar personal bien entrenado y calificado.

- Proporcionar excelente capacidad analítica, utilizando la instrumentación adecuada y los medios más eficientes a bajo costo.

- Utilizar procedimientos analíticos normalizados y actualizados para asegurar la producción de resultados exactos, precisos y confiables.

- Usar técnicas estadísticas y materiales de referencias certificados para el control de calidad en análisis de rutina.

- Efectuar revisiones inter-laboratorios con el fin de comprender y reducir las fuentes de variación de los procesos analíticos.

- Mantener la formación permanente del personal en el manejo de la calidad estadística y la actualización de nuevas metodologías con el fin de mejorar la calidad continuamente.

- Documentar todas las actividades del laboratorio: los procedimientos de gestión, los procedimientos técnicos, los resultados de las calibraciones, las instrucciones de trabajo, los registros. Es decir, documentar todo lo relacionado con el Sistema de la Calidad (SC) basado en la guía del sistema de gestión de la documentación ISO/TR 10013.

- Realizar auditorías internas para evaluar y mejorar el SC en el laboratorio a través de la implementación de las acciones correctivas, preventivas y revisión por parte de la Alta Dirección.

\subsection{La seguridad como elemento primordial}

Un elemento primordial para un laboratorio es asegurarse que su personal labora en un medio seguro. Este requisito está implícito en el numeral 4.1.2 de la norma ISO/IEC 17025:05. Las normas de seguridad en el manejo de reactivos, químicos, solventes gases y fuentes de radiación deben ser observadas cabalmente. El personal debe usar el equipo protector las veces que sea necesario.

El laboratorio debe tener un inventario de todos los químicos que ingresan, su cantidad y su lugar adecuado, así como la hoja de los datos de seguridad (del material). Se debe realizar un rápido chequeo (o revisión) de la calidad de todas las sustancias químicas que ingresan al laboratorio antes de agregarlos al inventario. Los residuos generales en el laboratorio deben ser eliminados de una manera aceptable al medio ambiente, seguro.

Los documentos se deben de mantener en forma segura para garantizar la confiabilidad y confidencialidad a los clientes. La vigencia de estos documentos debe ser por un período de tiempo de acuerdo a la dinámica propia del laboratorio o sugerido por los organismos reguladores.

\subsection{Personal}

El personal de laboratorio debe estar entrenado y calificado correctamente. Aunque, la mayoría de los empleados en los laboratorios están divididos en las categorías administrativas, profesionales y técnicas, el personal en general es el responsable de las operaciones con las más altas precauciones de seguridad del equipo del laboratorio.

Así mismo, es el responsable de mantener la seguridad de los datos y la información de los productos; es responsable del desempeño de los análisis, utilizando métodos, técnicas y patrones de referencia apropiados; debe mantener la calidad e integridad estadística de los datos: documentación de datos, métodos de ensayo, calibración y control de calidad; las cartas de control deben estar actualizadas para todas las pruebas 0 ensayos; el personal del laboratorio debe efectuar las acciones correctivas pertinentes; deben identificar las necesidades analíticas a largo plazo y desarrollo de planes que satisfagan esas necesidades; se debe conformar un equipo de evaluación de la conformidad 
en las actividades del laboratorio para mejorar el desempeño del laboratorio y dar seguimiento a los resultados de los ensayos Inter-laboratorios.

El personal del laboratorio debe estar familiarizado con los métodos de ensayo, la instrumentación y las prácticas de gestión de la calidad. El desempeño del personal nuevo recién entrenado debe demostrar su competencia antes de llevar a cabo el análisis para los clientes y cada laboratorio debe tener un programa de entrenamiento continuo para que el personal pueda mejorar su desempeño.

\subsection{Locales y condiciones ambientales apropiadas}

Las instalaciones y locales deben estar muy bien definidos de acuerdo al tipo de ensayo a realizar. Las secciones 0 áreas de trabajo deben ser funcionales teniendo en cuenta el riesgo de la contaminación cruzada. Se debe evitar la combinación de áreas incompatibles. Las condiciones ambientales, tales como flujo eléctrico, radiaciones, temperatura, humedad relativa, ruido, interferencias magnéticas, etc., deben ser apropiadas a los requisitos del método.

\subsection{Instrumentación y Reactivos}

Para cualquier ensayo, un laboratorio debe utilizar su propia instrumentación y debe tener un programa para reemplazar el equipo obsoleto. Debe existir un plan para el mantenimiento preventivo de rutina y registrar las reparaciones que se hagan. Una buena técnica es elaborar una bitácora para cada equipo e incluir su mantenimiento histórico.

Los equipos de laboratorio deben estar clasificados ${ }^{[19]}$ como: a) de servicio general no utilizados para realizar mediciones ni que tengan la mínima influencia en las mediciones (platos de calentamiento, agitadores, cristalería no volumétrica y cristalería utilizada para volúmenes aproximados tales como probetas, beakers, erlenmeyers) y sistemas de calentamiento y ventilación. b) material volumétrico tales como matraces, pipetas, picnómetros, buretas, etc. c) instrumentos de Medición, tales como espectrofotómetros, cromatógrafos, instrumentos electroquímicos, balanzas, viscosímetros, termómetros, hidrómetros, etc. d) referencias de mediciones físicas, masas certificadas, termómetros de referencia, etc. e) computadores y procesadores de datos. En cuanto a los reactivos, el laboratorio debe adquirir reactivos con garantía de calidad y verificarlos antes de ponerlos en uso. Los fabricantes o suplidores deben tener implantado un sistema de la calidad, el cual debe ser verificado por el laboratorio.

\subsection{Los ensayos}

Un laboratorio debe documentar completamente sus métodos de prueba. Los métodos de prueba requeridos deben conformarse con una metodología normalizada, tales como las establecidas por las ISO (Organización Internacional para la Normalización), ASTM (Sociedad Americana de Materiales y Ensayos), EPA (Agencia para la Protección del Medio Ambiente), AOAC (Organización Americana de Químicos Analíticos), AOCS (Sociedad Americana de Aceites y Grasas) y la FDA (Administración de Drogas y Medicamentos).

Los métodos no normalizados o normalizados modificados o desarrollados por el laboratorio deben ser validados por el personal competente del laboratorio. Si un método normalizado es utilizado por primera vez, el laboratorio debe verificarlo (confirmación de algunos parámetros de validación, según sea especificado en el método normalizado).

La validación consiste en evaluar los parámetros que caracterizan el desempeño del método, tales como: precisión, exactitud, límite de detección y de cuantificación, linealidad, sensibilidad y robustez del método. Una principal fuente de error es la desviación de los patrones o materiales de referencia debido a la contaminación accidental o intencional o a malas condiciones de manipulación y almacenamiento. El laboratorio debe tener un sistema que periódicamente revise la conformidad de estos materiales.

\subsection{Calibración}

Debe diseñarse un programa integral de calibración del equipo de medición para asegurarse que, donde el concepto sea aplicable, todas las mediciones sean trazables al Sistema Internacional (SI) a través de certificados que disponga el laboratorio, sea un patrón nacional o internacional o muestras de referencias certificadas.

Diferentes métodos de ensayo requieren diferentes calibraciones. Las decisiones sobre la calibración apropiada, así como su frecuencia, deben basarse en el análisis de caso por caso. Sin embargo, todas las pruebas analíticas requieren de la calibración, las cuales deben ser completamente documentadas. Si un instrumento no está calibrado, bajo ninguna circunstancia esos datos deben ser enviados al cliente.

\subsection{Trazabilidad}

La trazabilidad se define como la propiedad de un resultado de una medición o valor de un patrón que 
puede relacionarse a referencias declaradas con su incertidumbre asociada, usualmente patrones nacionales o internacionales, a través de una cadena ininterrumpida de comparaciones ${ }^{[8]}$.

El propósito de establecer la trazabilidad es asegurar que las mediciones, al final de la cadena de trazabilidad, pueda hacerse en unidades SI (Sistema Internacional). De esta manera, ellos son exactos y por tanto comparables con las mediciones realizadas en diferentes laboratorios, utilizando otros métodos.

\subsection{Materiales de Referencia y la trazabilidad}

Los materiales de referencia certificados (MRCs), patrones de referencia puros y muestras de referencia, son necesarios para el proceso de calibración o para el control de calidad ${ }^{[9]}$. Estos materiales se utilizan con mayor frecuencia en la calibración. Una de las principales fuentes donde se pueden adquirir los MRCs es en el Instituto Nacional de Normas y Tecnología (NIST) ${ }^{\text {[10] }}$ o en la BCR (Oficina de Referencias Certificadas) ${ }^{[11]}$ en Europa. La calibración de los MRCs deberían ser trazables a patrones nacionales y la trazabilidad debe ser preservada en el sistema de documentación del laboratorio.

Cuando no se disponen de patrones de referencia comerciales se pueden fabricar referencias internas. Estos patrones de referencia interna se pueden elaborar, tomándose una cantidad suficiente del material disponible en el laboratorio y se homogeniza. Luego se efectúa el análisis del componente de interés (analito) utilizando, en la medida de lo posible, métodos primarios clásico de análisis, tales como la volumetría, la gravimetría o culombimetría.

Los métodos tales como la espectrocopía de absorción atómica, la espectrocopía de emisión atómica de plasma acoplada inductivamente y la espectrometría de fluorescencia de rayos $X$ no pueden ser utilizada para certificar esos estándares de calibración primaria ya que son métodos que dependen de un proceso de calibración. Ellos pueden, sin embargo, ser útiles para confirmar datos obtenidos por los métodos primarios. Si es posible, se deben de utilizar dos métodos primarios diferentes. El tamaño de la muestra debe ser lo suficiente como representar la media poblacional.

Para obtener las muestras de referencia interna con matriz similar a la muestra a analizar, se debe tomar una cantidad razonablemente grande del producto disponible en el laboratorio o la planta de producción y homogenizar bien el material de tal forma que la muestra que se tome con referencia interna o de control represente el contenido del analito en la totalidad del material original. El valor de referencia debe obtenerse a partir de 20 a 30 repeticiones del análisis.

No es recomendable utilizar el mismo material de referencia utilizado como estándar para la calibración de la instrumentación que para el Control de Calidad de los análisis de las muestras. Este último debe tener una matriz similar al de la muestra a ser analizada. No obstante, esto no siempre es factible.

Cuando un MRC no está al alcance del laboratorio para utilizarlo en el proceso del control de calidad, basta tomar una muestra real analizarla 20 a 30 veces con un método de referencia, calcular el valor promedio y la incertidumbre para elaborar la carta de control. Es muy frecuente y más económico, elaborar muestras de referencia interna por adición de patrones referencia certificados a la muestra, homogenizar, analizar y calcular el porcentaje de recuperación o recobrado.

\subsection{Cartas de Control}

Algunos datos generados en el laboratorio deben ser rápidamente graficados en una carta de contro|[4], la cual consiste en representar en un gráfico los resultados de la realización periódica del análisis de un estándar o muestra de referencia. Los analistas quienes producen los datos - no el supervisor - son los que deben ubicar los resultados en la carta con el fin de promover el sentido de propiedad y fomentar el hábito de buscar la solución del problema. La carta debe estar ubicada en forma visible cerca del lugar de análisis para tenerla como referencia. La carta no debe ser considerada como terminal; el comportamiento estadístico debe verse continuamente y en el momento en que se obtengan datos fuera de los límites de control, los análisis de las muestras no deben ser enviadas al cliente. Se debe tomar una acción para remediar el problema y deben documentarse las causas de la anomalía y la forma en que fue solucionada.

En las plantas de fabricación es buena técnica tener dos cartas de control para el mismo análisis, una utilizando una muestra de referencia para medir la varianza del laboratorio y una segunda utilizando los productos de la planta día tras día, para medir la varianza del proceso. En un laboratorio bien controlado, la precisión analítica debe ser mucho mejor que la precisión del proceso. Eventualmente, la carta de control del proceso o laboratorio se convierte en parte del certificado y debe enviarse al cliente para promover su confianza en el laboratorio. Utilizando las cartas de control, un laboratorio podría calcular mensualmente o trimestralmente la precisión o incertidumbre para pruebas individuales. 
Estas incertidumbres comparadas con la de los métodos normalizados pueden proporcionar una visión clara del desempeño del método con respecto a normas industriales y detectar la necesidad de una acción correctiva. La revisión periódica debe ser una característica clave de cualquier programa de mejoramiento continuo.

\subsection{Aseguramiento del Control de la Calidad en las Mediciones (ACCM)}

El aseguramiento del control de la calidad en las mediciones ${ }^{[6]}$ consiste en analizar un estándar o material de referencia después de cada serie de análisis. Esto es, con el fin de garantizar el desempeño del método a través de la repetibilidad de los resultados (capacidad de obtener resultados muy cercanos entre sí cuando se realizan análisis repetidos).

Es sorprendente, cómo la mayoría de los buenos laboratorios no practican el ACCM. Este proceso debe ser visto como parte integral de una secuencia analítica completa, y no como un proceso adicional innecesario. Una programación escrita (un calendario de actividades) debe ser elaborada para el ACCM frecuentemente, y todo el personal de laboratorio debe de familiarizarse con él. Con frecuencia, la práctica ACCM, depende del tipo de muestra y del instrumento; ciertamente, al menos un patrón de referencia debe ser analizado con cada lote de muestras del cliente.

Un diseño posible para el ACCM puede ser el siguiente: un patrón de referencia debe ser analizado antes y después de una serie de resultados; el análisis de una muestra de referencia (o muestra control) deben interponerse con varias muestras del cliente (lote) para monitorear continuamente la calidad de los datos analíticos. Una regla general es analizar una muestra control (como de referencia) por cada 5 a 10 muestras. Si el análisis de la muestra control indica resultados incongruentes, ningún dato debe ser enviado al cliente. Se debe tomar una acción correctiva inmediata para remediarlo.

\subsection{Evaluación de la incertidumbre de la medición (IDM)}

En los métodos cuantitativos se debe evaluar la incertidumbre de los resultados, siguiendo la guía ISO GUM 1995:08[12] y GUM 2008 (Suplemento 1) ${ }^{[13]}$. Cada laboratorio debe tener un procedimiento para la evaluación de la incertidumbre. La incertidumbre $\left(u_{y}\right)$ se define como un parámetro asociado al resultado de una medición $(y)$, que caracteriza la dispersión de los valores que pudieran ser atribuidos al mensurando (cantidad sujeta a medición: masa, volumen, mol, temperatura, corriente, absorbancia, etc.) basado en la información utilizada $^{[8]}$.

La incertidumbre describe un rango en el cual existe la oportunidad razonable de encontrar un valor que puede ser apropiadamente atribuida al mensurando ${ }^{[14]}$. En el cálculo de la incertidumbre, es importante tener en cuenta no sólo la medición instrumental sino también las diferentes etapas del ensayo. Todo laboratorio que realiza ensayos cuantitativos debe reportar los resultados con su incertidumbre asociada. Por ejemplo, un resultado del análisis de plomo en sangre de un paciente, debe reportarse así: $y \pm U$. Donde y es el mensurando o valor de plomo en $\mu \mathrm{g} / \mathrm{dL}$ de sangre total y $U$ es la incertidumbre $u_{y}$ multiplicada por un factor $k$, denominada factor de cobertura, cuyo valor está basado en el tipo de distribución (generalmente normal) con un nivel de probabilidad determinado. Entre menor sea la incertidumbre, mayor es la precisión del método.

\subsection{Ensayos inter-laboratorios}

Los ensayos de colaboración o revisiones cruzadas Inter-laboratorio ayudan a medir la capacidad de un laboratorio con respecto a sus competidores. Cuando un laboratorio es menos preciso que otro, la causa debe ser buscada y eliminada. En caso de reclamos por parte del cliente o proveedor, la calidad de los datos obtenidos de las revisiones cruzadas, muestran al cliente que el laboratorio está trabajando con la calidad esperada. Esto dará mayor confianza al cliente o suplidor. La participación en una revisión cruzada como parte del mejoramiento continúo significa estudiar los datos y corregir las deficiencias.

La revisión cruzada debe ser con materiales estables y homogéneos. Las precauciones deben asegurar que el material no se degradará o descompondrá entre el despacho y la recepción. En una revisión cruzada se debe incluir un número suficiente de laboratorios y muestras para obtener conclusiones estadísticamente significativas. Cualquier laboratorio puede participar en programas de ensayos inter-laboratorios organizados por diferentes organizaciones especializadas. Así por ejemplo, la American Society for Testing and Materials ASTM $^{[15]}$ y la American Oficial of the Analytical Chemist $A O A C^{[16]}$, recomienda al menos ocho laboratorios. Los datos obtenidos deben someterse a un proceso de análisis estadístico para comprobar la existencias de puntos "outliers" o datos aberrantes, los cuales deben desecharse antes de calcular la repetibilidad intralaboratorio y la reproducibilidad interlaboratorio. 
Además de la tabla de datos, la representación gráfica es de gran utilidad para una sinopsis rápida del desempeño de un laboratorio en el proceso de la revisión cruzada. Es importante señalar que, un outlier (dato fuera de lugar o valor dudoso o aberrante) obtenido en un laboratorio debe aplicar una acción inmediata para estudiar las causas de la anomalía y luego aplicar una acción correctiva y establecer un procedimiento para la aplicación de una acción preventiva, e implementar las mejoras pertinentes.

\subsection{Subcontrataciones}

Algunas veces se vuelve necesario la subcontratación de otro laboratorio, como por ej.: cuando el trabajo requiere un instrumento en particular que el laboratorio no posee. Es importante asegurarse que el laboratorio subcontratado tenga implementado el Sistema de la Calidad. Con el fin de comprobar el Sistema de Calidad del laboratorio contratado, es recomendable enviar una "muestra ciega o testigo" (muestra cuyo contenido del analito se conoce con certeza) junto con la muestra real; si los resultados son congruentes, se puede aceptar que los resultados de la muestra son confiables. El laboratorio subcontratado debe proporcionar un certificado de análisis.

\subsection{Sistema de gestión de la información LIMS}

La mayoría de los laboratorios modernos utilizan algún tipo de sistema de gestión de la información de laboratorio computarizado (LIMS), un número de los cuáles están disponibles comercialmente. Las capacidades mínimas de tales sistemas incluyen lo siguiente:

- Producción de datos de muestras pendientes.

- Control de las muestras del cliente para su conteo.

- Archivar datos históricos sobre calibraciones o Control de Calidad.

- Recordar cuando se hicieron las calibraciones y los Controles de Calidad.

- Controlar los datos de Control de Calidad. El programa muestra las desviaciones a las reglas estadísticas cuando ellas ocurren.

- Calcular la precisión del laboratorio de forma periódica para varias pruebas.

- Comparar los análisis contra las especificaciones del producto; si es relevante.

- Proporcionar bases de datos históricas para ensayos y productos.

- Proporcionar, cuando sea posible, una relación de los datos automatizados con el tipo de instrumento de laboratorio.
La administración no debería ver al sistema LIMS como una panacea que resuelve los problemas; el sistema es una herramienta para ordenar y almacenar datos de laboratorio y registrarlos. El personal del laboratorio debe garantizar la manera de mejorar continuamente los métodos, la precisión y la exactitud.

\subsection{La documentación}

Cada laboratorio debe tener un manual de la calidad (MC) que describa las políticas de la calidad y las actividades generales del laboratorio, haciendo referencias a procedimientos de gestión e instrucciones de trabajo. Debe tener documentada la organización y proporcionar con detalles las responsabilidades del personal, políticas y programas de entrenamiento del personal, lista de equipos, un programa y calendario de mantenimiento de la instrumentación, políticas de adquisición (compras), inventario de reactivos, un plan de la calidad, un programa de calibración y calendarización, la frecuencia del Contro de Calidad con el uso de materiales de referencia (interna MRI y certificados MRC) y copias de métodos de análisis o ensayos. Todos estos documentos deben estar organizados en una estructura piramidal ISO/TR $10013^{[17]}$, iniciando por el MC en la cúspide, seguido de los procedimientos de gestión, procedimientos técnicos, instrucciones de trabajo y registros.

\subsection{Certificado de los análisis}

El laboratorio necesita emitir un certificado de los análisis para transmitir los datos de los análisis a los clientes. Los componentes principales de este certificado son: el nombre y dirección del laboratorio; nombre del cliente y su dirección; número de referencia para el reporte; identificar las muestras de los clientes y el número de identificación del laboratorio; indicación del método de ensayo, los resultados con unidades de medidas en sistema internacional (SI), incertidumbre de las mediciones si es relevante; detalles acerca del análisis si es relevante, una firma autorizada y fecha de transmisión de los datos. Internamente un certificado de análisis debe ser completamente trazable a la calibración, el Control de Calidad y cualquier otra información apropiada.

\subsection{Certificación ISO 9000 y la Acreditación}

El modelo de gestión de la calidad de la familia de normas ISO 9000, se fundamenta entre otros principios, en:

- La planificación de todas las actividades relativas a la calidad. 
- Las acciones correctivas que garantiza la no recurrencia de las no conformidades.

- Las acciones preventivas que eviten la ocurrencia de no conformidades potenciales.

- El mejoramiento continuo.

- Una mayor conciencia de la calidad en la organización.

- La claridad de las distribuciones de responsabilidades.

- Mayor eficacia interna.

- La disciplina, constancia y regularidad en las tareas. ${ }^{[17]}$

- Un mayor compromiso y participación de los miembros de la organización en las metas de la calidad.

Todos estos principios o lineamientos, en los que se fundamenta la familia de las normas ISO 9000 hacen que todos y cada uno de los esfuerzos de los miembros de una organización estén eficazmente coordinados y orientados, a necesidades y expectativas, a menor costo y más eficazmente. Es decir, aumenta el grado de satisfacción de los clientes. En la década de los ochenta, el registro de los sistemas de calidad ISO 9001 se convirtió en una forma de vida de las compañías y laboratorios a través de todo el mundo. Ciertamente es un medio para documentar sistemáticamente todas las actividades de laboratorio. Sin embargo, esto debe verse como la base fundamental para construir un sistema TQM verdadero.

Los laboratorios de ensayos y análisis se rigen por requisitos de gestión establecidos en la norma ISO/ IEC 17025:2005 y no se contradicen con la norma ISO 9001:2008. Los requisitos de gestión de las dos normas son equivalentes. A nivel internacional existen organismos de acreditación de laboratorio, tales como la Asociación Americana para la Acreditación de Laboratorio (A2LA) en Estados Unidos, y el Comité Francés para la Acreditación (COFRAC) en Francia. En Nicaragua existe la OFICINA NACIONAL PARA LA ACREDITATACION (ONA) adscrita al Ministerio de Fomento, Industria y Comercio (MIFIC), cuyas normas son las NTN 04-001 05. La meta de un laboratorio es obtener la acreditación con el fin de garantizar la credibilidad ante los clientes.

No se debe confundir la Acreditación con la Certificación ISO 9001. Las dos están basadas en normas diferentes. La Certificación es la declaración por parte de una autoridad o un organismo calificado de que el sistema de gestión de una organización o empresa cumple con los criterios definidos en las normas ISO 9001.
La acreditación es el reconocimiento formal que extiende un organismo competente (ONA-MIFIC) declarando que el laboratorio es técnicamente competente para realizar ensayos y/o calibraciones específicos, basado en normas técnicas voluntarias como la guía ISO/IEC 17025:2005 o la NTN 04-001 05 ${ }^{[18]}$. IEC son las siglas de la Comisión Internacional de Electricidad; NTN significa Norma Técnica Nicaragüense. Es importante recalcar que cuando se implementa un Sistema de la Calidad en un laboratorio, se debe pensar en una inversión en la que se obtendrá valor agregado por las siguientes razones:

- Se minimizan los reclamos.

- Se evitan repeticiones de los análisis.

- Se racionaliza el uso de materiales y reactivos.

- Se minimiza el tiempo de análisis.

- Se evita el deterioro de los instrumentos.

\subsection{Auditorías internas}

Todo laboratorio debe tener un sistema de revisión periódica de sus propias actividades para confirmar la concordancia con los sistemas documentados. Aún si el laboratorio está sometido a una auditoría externa bianual, es importante efectuar auditorias internas anuales (o especiales cuando lo amerite el caso) de acuerdo a un plan y un programa bien definidos. Los evaluadores (o Auditores) internos están más familiarizados con los requerimientos de laboratorio que los auditores del organismo de Acreditación. En esas auditorías el laboratorio debe revisar si cumple con su propio sistema de calidad y si las pruebas se están realizando correctamente.

Los informes y recomendaciones de los auditores internos deben ser revisados por el director del laboratorio y actuar sobre las no conformidades encontradas aplicando las correcciones correctivas respectivas e implementar las mejoras con enfoque a la satisfacción del cliente. De esta forma, el laboratorio está implementando la mejora continua y se está construyendo su propia fortaleza. A través de las auditorías se corrigen las deficiencias, se fortalece el laboratorio y la empresa o laboratorio adquiere mayor calidad para poder satisfacer a sus clientes.

\subsection{Otras prácticas de calidad}

Además de los protocolos o procedimientos normalizados en torno a los ensayos, el laboratorio puede elaborar y aplicar protocolos estadísticos. Estos protocolos incluyen situaciones tales como: muestras representativas, aceptabilidad de ensayos repetidos, divergencias de la calidad de los datos entre laboratorios, 
aceptación de datos contra las especificaciones del producto y uso de cifras significativas. En tales situaciones un laboratorio debe aplicar estos protocolos en forma congruente, para poder tomar decisiones correctas de mejora.

\section{CONCLUSIONES}

De todo lo anterior, se puede deducir que la modernización de un laboratorio de servicio está basada en un sistema de actividades cuyo propósito es el de asegurar la calidad de los servicios de ensayos (analíticos, microbiológicos o biológicos) de acuerdo a normas de calidad definidos en normas nacionales e internacionales. Esto significa, implantar un Sistema de la Calidad que normalice todas las actividades del laboratorio a través de procedimientos documentados y la generación de registros que garanticen la trazabilidad documental y metrológica de los servicios que brinda la organización o laboratorio a los clientes.

Este Sistema deberá ser reconocido por un organismo competente a través de la acreditación, la cual está basada en el cumplimiento de una serie de requisitos establecidos por la norma internacional ISO/IEC 17025:05 o su equivalente en las Normas Técnicas Nicaragüense NTN 04-001 05 . Un laboratorio Acreditado, tiene el reconocimiento nacional e internacional de que sus resultados de ensayos cumplen con la calidad requerida de los clientes: exactitud, precisión y confiabilidad.

\section{REFERENCIAS BIBLIOGRÁFICAS}

1. DEMING E., (2000), "Out of the Crisis". First MIT Press Edition, Massachussets, pag. 18

2. JAMES P., (2001), Gestión de la Calidad Total, Prentice Hall, España. Pag. 45.

3. JURAN J.M., GRYNA F.M., (1998), Análisis y planeación de la calidad, 3a Ed., McGraw Hill, México, pag. 150.

4. ISHIKAWA, K., (1976)," Guía para el control de la Calidad", Organización Asiática para la Publicidad, pag. 214.

5. ISO 9000:2005 (ES), (2005), Sistemas de Gestión de la Calidad. Fundamentos y Vocabulario

6. ISO/IEC 17025(ES) (2005). "Requisitos Generales para la Competencia de los Laboratorios de Ensayo y de Calibración", Norma Internacional, Ginebra, Segunda Edición.
7. ISO 9000:2005 (E), (2008),Sistemas de Gestión de la Calidad-Requisitos. Norma Internacional, Ginebra.

8. VIM (2008). JCCM Comité Conjunto de Metrología International vocabulary of metrology.

9. ILAAC G9:2005, Guía para el uso de materiales de referencia. Acceso en Internet: http://www. organismouruguayodeacreditacion.org/IAAC

10. NIST, http://ts.nist.gov/measurementservices/ referencematerials/index.cfm

11. IRMN, Institute for Reference Materials and Measurements, http://www.irmm.jrc.be. Comercializa los MRCs de la BCR.

12. BIPM, IEC, IFCC, IUPAC, OIML (2008). Guide for to the Expression of Uncertainty in Measurement (GUM), ISO, Ginebra.

13. BIPM, IEC, IFCC, IUPAC, OIML (2008). Guide for to the Expression of Uncertainty in Measurement (GUM). Supplement 1, ISO, Ginebra.

14. HIBBERT, B. (2007), Quality Assurance in the Analytical Chemistry Laboratory, Oxford Press, pag. 163

15. ASTM's Proficiency test programs on Chemical Analysis of Metals. En http://www.brammerstandard. com/pdf/astm.ptp.pdf, acceso el 14/10/09.

16. AOAC's Proficiency Testing Program Scores HighReceives Accreditation by A2LA http://www. aoac.org/proficiencytesting/profTestingPrograms. html\#Pesticide. Acceso el 14/10/09.

17. ISO/TR 10013:2001 (E), (2001), "Guidelines for quality management system documentacion.

18. NTN 04-001 05, (2005), "Requisitos generales para la competencia de los laboratorios de ensayos y de calibración. Norma nicaragüense.

19. EURACHEM/CITAC, (2002), Guide to Quality in Analytical Chemistry 